\title{
Intensification of older adults' outpatient blood pressure treatment at hospital discharge: national retrospective cohort study
}

\author{
Timothy S Anderson, ${ }^{1}$ Charlie M Wray, ${ }^{2}$ Bocheng Jing, ${ }^{3}$ Kathy Fung, ${ }^{3}$ Sarah Ngo, ${ }^{3}$ Edison Xu, ${ }^{3}$ \\ Ying Shi, ${ }^{3}$ Michael A Steinman ${ }^{4}$
}

${ }^{1}$ Division of General Internal

Medicine, University of

California San Francisco, San

Francisco, CA 94123, USA

${ }^{2}$ Division of Hospital Medicine, University of California San Francisco, San Francisco,

CA, USA

${ }^{3}$ San Francisco Veterans Affairs Medical Center, San Francisco,

CA, USA

${ }^{4}$ Division of Geriatrics, University of California San

Francisco, San Francisco,

CA, USA

Correspondence to:

TS Anderson

timothy.anderson@ucsf.edu (or @TimAndersonMD on Twitter; ORCID 0000-0002-5384-275X)

Additional material is published online only. To view please visit the journal online.

Cite this as: $B M J$ 2018;362:k3503 http://dx.doi.org/10.1136/bmj.k3503

Accepted: 26 July 2018

\section{ABSTRACT}

\section{OBJECTIVES}

To assess how often older adults admitted to hospital for common non-cardiac conditions were discharged with intensified antihypertensive treatment, and to identify markers of appropriateness for these intensifications.

DESIGN

Retrospective cohort study.

SETTING

US Veterans Administration Health System.

\section{PARTICIPANTS}

Patients aged 65 years or over with hypertension admitted to hospital with non-cardiac conditions between 2011 and 2013.

\section{MAIN OUTCOME MEASURES}

Intensification of antihypertensive treatment, defined as receiving a new or higher dose antihypertensive agent at discharge compared with drugs used before admission. Hierarchical logistic regression analyses were used to control for characteristics of patients and hospitals.

RESULTS

Among 14915 older adults (median age 76, interquartile range 69-84), 9636 (65\%) had well controlled outpatient blood pressure before hospital admission. Overall, 2074 (14\%) patients were discharged with intensified antihypertensive

\section{WHAT IS ALREADY KNOWN ON THIS TOPIC}

More than half of adults admitted to hospital are discharged with changes to multiple outpatient drug treatments

Transient elevations of blood pressure are common in adults admitted to hospital

Whether hospital admission leads to intensification of older adults' outpatient blood pressure drug regimens is unknown

\section{WHAT THIS STUDY ADDS}

In this retrospective cohort study of patients admitted to US Veterans

Affairs hospitals, one in seven older adults were discharged with intensified

antihypertensive drugs

More than half of these patients had previously well controlled outpatient blood pressure

Elevated inpatient blood pressure strongly predicted intensification, even in patients with previously well controlled outpatient blood pressures or a low likelihood of benefit from strict blood pressure control

More attention is needed to reduce potentially harmful overtreatment of blood pressure as older adults transition from hospital to home

treatment, more than half of whom (1082) had well controlled blood pressure before admission. After adjustment for potential confounders, elevated inpatient blood pressure was strongly associated with being discharged on intensified antihypertensive regimens. Among patients with previously well controlled outpatient blood pressure, $8 \%$ (95\% confidence interval $7 \%$ to $9 \%$ ) of patients without elevated inpatient blood pressure, $24 \%$ (21\% to $26 \%$ ) of patients with moderately elevated inpatient blood pressure, and $40 \%$ (34\% to $46 \%$ ) of patients with severely elevated inpatient blood pressure were discharged with intensified antihypertensive regimens. No differences were seen in rates of intensification among patients least likely to benefit from tight blood pressure control (limited life expectancy, dementia, or metastatic malignancy), nor in those most likely to benefit (history of myocardial infarction, cerebrovascular disease, or renal disease).

\section{CONCLUSIONS}

One in seven older adults admitted to hospital for common non-cardiac conditions were discharged with intensified antihypertensive treatment. More than half of intensifications occurred in patients with previously well controlled outpatient blood pressure. More attention is needed to reduce potentially harmful overtreatment of blood pressure as older adults transition from hospital to home.

\section{Introduction}

More than half of adults admitted to hospital are discharged with changes to four or more of their outpatient drug treatments. ${ }^{1-3}$ Although substantial efforts have been made to manage and communicate changes in drug treatment at the time of discharge from hospital (for example, medication reconciliation), comparatively little focus has been placed on the appropriateness of the changes themselves. ${ }^{45}$ Even when clinically indicated, drug changes create risks to patients including adverse drug events, medication confusion, and drug interactions. When drug changes made during hospital admission and continued at discharge are non-essential, they may cause more harm than good, exposing patients to potentially serious harms with minimal likelihood of benefit. ${ }^{6-8}$

One area of particular importance is the modification of longstanding regimens for chronic diseases that are not directly related to hospital admission. ${ }^{6}$ These considerations are particularly crucial for hypertension, as blood pressure is frequently measured during hospital admission and transient fluctuations 
are common. Despite a lack of evidence indicating benefit from strict inpatient blood pressure control in most clinical scenarios, elevated readings may lead clinicians to intensify antihypertensive treatment regimens. ${ }^{910}$ Strict control of chronic diseases during the hospital stay can easily become overtreatment when continued on discharge and expose the patient to increased treatment burden and a higher risk of serious adverse events. ${ }^{1011}$ Furthermore, in modifying drugs for chronic diseases, inpatient clinicians may not be aware of important contextual factors, such as history of previous drug treatment, drug intolerance, barriers to adherence, and the patient's long term success at disease control. ${ }^{12}$

Previous research has not examined how frequently antihypertensive drugs are intensified during hospital admission and continued at discharge or which patients are most likely to have their drugs changed. We examined a national cohort of older adults with hypertension who wereadmitted to hospital for common medical conditions to assess how often outpatient antihypertensive agents are intensified during hospital admission, to what extent inpatient blood pressure recordings and outpatient blood pressure control are driving intensification of antihypertensive treatment, and whether chronic comorbidities influence decisions to intensify treatment during hospital admission.

\section{Methods}

Data source and study population

We did a retrospective cohort study of older adults admitted to hospital by using national inpatient and outpatient Veterans Affairs pharmacy data merged with Veterans Affairs and Medicare claims data from fiscal years 2009-14. The Veterans Affairs health system is a national integrated health system in the US that provides care to more than 9 million US veterans at more than 1200 outpatient clinics and 170 medical centers. Inpatient care at the Veterans Affairs is provided by a combination of attending physicians who specialize in inpatient medicine (hospitalists) and primary care physicians and specialists who intermittently attend on inpatient services. The Veterans Affairs health system is a primary site for graduate medication education in the US, so many patients will be cared for by a team of trainees (medical students and residents) and an attending physician. The study cohort consisted of all patients aged 65 years and older with a diagnosis of hypertension who received regular Veterans Affairs outpatient care and were admitted to a Veterans Affairs hospital between January 1, 2011 and December 31, 2013 for pneumonia, urinary tract infection, or venous thromboembolism. We chose these three conditions as they are common reasons for hospital admission and their treatment does not typically require aggressive blood pressure management.

We defined hypertension as at least two outpatient diagnoses or one hospital discharge diagnosis of hypertension in the two years preceding the index hospital admission. ${ }^{13}{ }^{14}$ We identified hospital admissions for pneumonia, urinary tract infection, and venous thromboembolism by primary discharge diagnosis ICD-9 (international classification of diseases, version 9) codes. ${ }^{13}$ We excluded patients with secondary discharge diagnoses that might necessitate an acute change in antihypertensive treatment (for example, atrial fibrillation or acute cardiovascular or cerebrovascular events). To ensure accurate capture of drug use, we limited our sample to patients who received at least $80 \%$ of their outpatient care in Veterans Affairs settings and thus were expected to regularly receive drugs from Veterans Affairs pharmacy sources, and we excluded patients admitted from or discharged to an institutional setting and those admitted to hospital in the preceding 30 days. Detailed exclusion criteria are depicted in supplementary figure A.

\section{Identifying drug intensifications}

Our primary outcome was whether a patient received one or more intensified outpatient antihypertensive agents at hospital discharge. We examined major antihypertensive drug classes, as identified by the seventh report of the Joint National Committee (JNC-7), released in 2004 and the primary blood pressure guideline in use during our study period. ${ }^{15}$ Drug classes included angiotensin converting enzyme inhibitors, angiotensin receptor blockers, $\beta$ blockers, calcium channel blockers, thiazide diuretics, centrally acting $\alpha 2$ agonists, direct vasodilators, aldosterone receptor blockers, and potassium sparing diuretics. We also included direct renin inhibitors, which were approved for treatment of hypertension after the publication of JNC-7. We excluded loop diuretics and $\alpha 1$ blockers, as they are more commonly used to treat edema and benign prostatic hypertrophy, respectively.

To establish whether a patient's antihypertensive drugs were intensified, we first established a list of admission and discharge antihypertensives for each patient. On the basis of validation work, described below, we defined active use at time of admission as the latest pharmacy fill being for a quantity sufficient to last until at least 60 days before hospital admission. This type of grace period is a commonly used criterion to account for carryforward of previously stockpiled drugs and transient non-adherence. ${ }^{16}{ }^{17}$ Next, we established a list of discharge drugs that included prescriptions filled by the outpatient pharmacy between two days before and two days after discharge, prescriptions filled by the inpatient pharmacy on the date of discharge with a supply of at least seven days, and drugs present on admission for which the patient had an adequate supply at home according to preadmission dispensing history.

We classed antihypertensives not present on the admission drug list but prescribed at discharge as new drug additions. We defined dose increases as admission drugs for which a discharge prescription was filled for a dose increase of more than $20 \%$. We classed both dose increases and the addition of new drugs as treatment intensifications. We then classified 
all patients as receiving an intensification if they received at least one dose increase or addition.

\section{Validation of identification of intensifications}

Pharmacy records cannot be used to reliably assess which drugs present on admission were not continued at discharge. Thus some patients with intensification of one or more antihypertensive drugs may also have had other antihypertensives stopped, resulting in net balance or deintensification of treatment for that patient and misidentification by our measure. To confirm the accuracy of our approach to using pharmacy records for identifying drug intensifications, we did a two stage chart review of 234 charts identified from stratified random samples of the study cohort (see supplementary methods and supplementary tables $A$ and $B)$. We found that patient level intensifications identified by pharmacy records had a sensitivity of $81 \%$ (95\% confidence interval 58\% to $95 \%$ ), a specificity of $96 \%$ ( $91 \%$ to $98 \%$ ), and a positive predictive value of $73 \%(63 \%$ to $81 \%)$.

\section{Covariates}

Our primary predictor variables were inpatient and outpatient blood pressure control. Outpatient blood pressure control was measured using the median of the three most recent readings recorded at outpatient visits before the seven days preceding hospital admission. We categorized outpatient blood pressure control as well controlled if systolic blood pressure was below 140 $\mathrm{mm} \mathrm{Hg}$ and diastolic blood pressure was below $90 \mathrm{~mm}$ $\mathrm{Hg}$, high if systolic pressure was 140-179 mm $\mathrm{Hg}$ and diastolic pressure was $90-99 \mathrm{~mm} \mathrm{Hg}$, or very high if systolic pressure was $180 \mathrm{~mm} \mathrm{Hg}$ or higher or diastolic pressure was $100 \mathrm{~mm} \mathrm{Hg}$ or higher. We categorized inpatient blood pressure control as severely elevated if three or more recordings of systolic blood pressure above $180 \mathrm{~mm} \mathrm{Hg}$ were measured during hospital admission, moderately elevated if three or more recordings of systolic pressure were above $160 \mathrm{~mm} \mathrm{Hg}$, or not elevated. Ninety six per cent (14347/14915) of patients had both inpatient and outpatient blood pressure values available.

Demographic and chronic disease covariates included age, sex, ethnicity, median household income estimated from residential zip codes, the Deyo adaptation of the Charlson comorbidity index (calculated from Veterans Affairs and Medicare claims during the 24 months before hospital admission), ${ }^{18}$ and individual constituents of that index. Hospital admission related covariates included year of admission, training hospital status, length of stay, and primary discharge diagnosis.

\section{Statistical analysis}

We did multivariable mixed effect logistic regression analyses to determine associations between the outcome of antihypertensive intensification and primary predictors of outpatient and inpatient blood pressure control. Adjusted analyses included the covariates noted above, a random effect term to account for clustering by hospital, and an interaction term to account for the relation between inpatient and outpatient blood pressure categories.

We then examined whether specific comorbidities and life expectancy affected the predicted probability of being discharged with intensified antihypertensives. We hypothesized that patients with chronic comorbidities associated with a greater potential benefit from strict blood pressure control (previous history of congestive heart failure, acute myocardial infarction, renal disease, and cerebrovascular disease) would be more likely to be discharged with intensified regimens, whereas patients with comorbidities associated with lower potential benefit (metastatic malignancy and dementia) and those with limited life expectancy (based on age and Charlson score $)^{1819}$ would be less likely to be discharged with intensified regimens. For this analysis, we again did multivariable logistic regression analyses using the approach described above but separating the Charlson index into dichotomous component variables for each comorbidity.

Patients with a history of congestive heart failure or acute myocardial infarction may haveantihypertensives such as $\beta$ blockers and angiotensin converting enzyme inhibitors adjusted for reasons other than blood pressure control, so we did two sensitivity analyses. Firstly, we calculated the proportion of patients receiving intensifications after restricting our cohort to patients without a secondary discharge diagnosis of congestive heart failure or past medical history of congestive heart failure or acute myocardial infarction. Secondly, we repeated our primary analysis but added indicator variables for a secondary discharge diagnosis or comorbidity of congestive heart failure and comorbidity of acute myocardial infarction.

We determined statistical significance by using 95\% confidence intervals and two tailed tests with $\mathrm{P}<0.05$. We used post-estimation margins to calculate predicted probabilities of intensification by blood pressure group, by comorbidity, and by estimated life expectancy. We used Stata version 14.1 for all analyses.

\section{Patient and public involvement}

No patients were involved in setting the research question or the outcome measures, nor were they involved in developing plans for design or implementation of the study. No patients were asked to advise on interpretation or writing up of results. As this study concerned examination of existing administrative claims data for a national sample of patients, no participants were specifically recruited for this analysis and there are no plans to directly disseminate the results of the research to study participants.

\section{Results}

\section{Cohort characteristics}

Table 1 shows the characteristics of the 14915 patients. The cohort was almost entirely male, the 


\begin{tabular}{|c|c|}
\hline Characteristic & Value $(n=14915)$ \\
\hline \multicolumn{2}{|l|}{ Demographics and chronic conditions } \\
\hline Median (interquartile range) age, years & $77(66-84)$ \\
\hline Male sex & $14437(96.8)$ \\
\hline \multicolumn{2}{|l|}{ Ethnicity: } \\
\hline White & $11486(77.0)$ \\
\hline Black & $2669(17.9)$ \\
\hline Hispanic & $277(1.9)$ \\
\hline Other/unknown & $482(3.2)$ \\
\hline Median (interquartile range) income, $\$$ & $43965(35192-55919)$ \\
\hline \multicolumn{2}{|l|}{ Charlson comorbidity score: } \\
\hline 0 (good health) & $872(5.9)$ \\
\hline 1-3 (average health) & $6154(41.3)$ \\
\hline$\geq 4$ (poor health) & $7889(52.9)$ \\
\hline \multicolumn{2}{|l|}{ Selected comorbidities: } \\
\hline Previous history of myocardial infarction & $1795(12.0)$ \\
\hline Congestive heart failure & $3980(26.7)$ \\
\hline Cerebrovascular disease & $3484(23.4)$ \\
\hline Renal disease & $5261(35.3)$ \\
\hline Dementia & $1004(6.7)$ \\
\hline Malignancy & $4802(32.2)$ \\
\hline Metastatic malignancy & $964(6.5)$ \\
\hline \multicolumn{2}{|l|}{ Pre-admission drug use } \\
\hline No (interquartile range) of drugs on admission & $7(4-11)$ \\
\hline No (interquartile range) of antihypertensive drugs on admission & $1(1-2)$ \\
\hline \multicolumn{2}{|l|}{ Antihypertensive drugs on admission: } \\
\hline Angiotensin converting enzyme inhibitors & $5756(38.6)$ \\
\hline Angiotensin receptor blockers & $1403(9.4)$ \\
\hline$\beta$ blockers & $6716(45.0)$ \\
\hline Calcium channel blockers & $4605(30.9)$ \\
\hline Thiazide diuretics & $3059(20.5)$ \\
\hline Other antihypertensives* & $1480(9.9)$ \\
\hline \multicolumn{2}{|l|}{ Hospital admission } \\
\hline \multicolumn{2}{|l|}{ Year of admission: } \\
\hline 2011 & $5547(37.2)$ \\
\hline 2012 & $4834(32.4)$ \\
\hline 2013 & $4534(30.4)$ \\
\hline \multicolumn{2}{|l|}{ Primary discharge diagnosis: } \\
\hline Pneumonia & $7726(51.8)$ \\
\hline Urinary tract infection & $5639(37.8)$ \\
\hline Venous thromboembolism & $1550(10.4)$ \\
\hline Training hospital & $13194(88.5)$ \\
\hline Median (interquartile range) length of stay, days & $4(3-6)$ \\
\hline
\end{tabular}

median age was 76 years, and most patients had multiple comorbidities. Half of the cohort had a primary discharge diagnosis of pneumonia $(n=7726)$, $38 \%$ urinary tract infection $(n=5639)$, and $10 \%$ venous thromboembolism $(n=1550)$. Patients were prescribed a median of one antihypertensive drug on admission.

Inpatient and outpatient blood pressure control Figure 1 shows the relation between inpatient and outpatient blood pressure control. Before admission, $65 \%(n=9639)$ of patients had well controlled outpatient blood pressure, 32\% (4567) had high outpatient blood pressure, and 2\% (287) had very high outpatient blood pressure. During hospital admission, $19 \%$ (2755) of patients had moderately elevated inpatient blood pressure and 5\% (775) had severely elevated inpatient blood pressure. Elevated inpatient blood pressure was more common in patients with

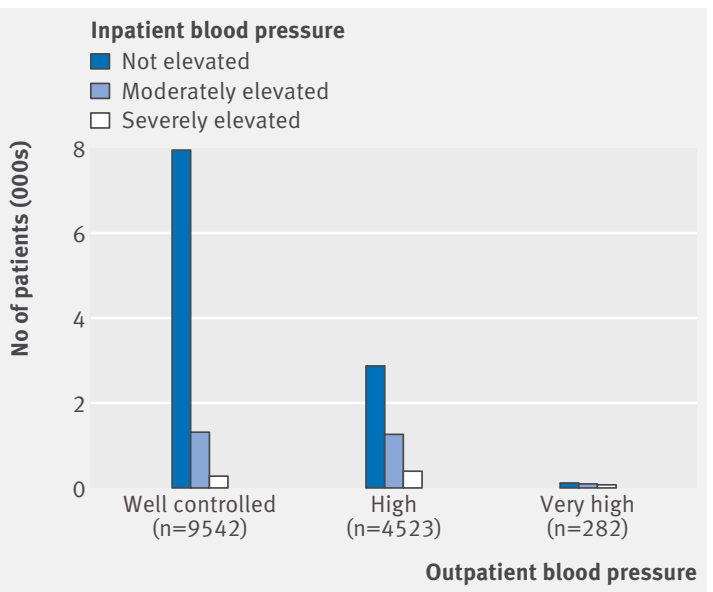

Fig 1 | Relation between inpatient and outpatient blood pressure (BP) recordings. Outpatient BP control measured using median of three outpatient BPs before admission and defined as well controlled if systolic blood pressure (SBP) $<140 \mathrm{~mm} \mathrm{Hg}$, high if SBP $140-179 \mathrm{~mm} \mathrm{Hg}$ or diastolic blood pressure (DBP) $90-100 \mathrm{~mm} \mathrm{Hg}$, or very high if $S B P \geq 180 \mathrm{~mm} \mathrm{Hg}$ or $D B P>100 \mathrm{~mm} \mathrm{Hg}$. Inpatient $B P$ control defined by number of elevated $B P$ recordings and defined as severely elevated if $\geq 3$ recordings of $S B P>180 \mathrm{~mm} \mathrm{Hg}$, moderately elevated if $\geq 3$ recordings of $\mathrm{SBP}>160 \mathrm{~mm} \mathrm{Hg}$ without meeting criteria for severely elevated, or not elevated

chronically elevated outpatient blood pressure before admission $(\mathrm{P}<0.001)$; however, 47\% (2399/5062) of the cohort with elevated inpatient blood pressure were normotensive before admission.

\section{Intensification of antihypertensives}

A total of 2074 (14\%) patients were discharged with an antihypertensive intensification; 1293 (9\%) were started on one new antihypertensive on discharge and 300 (2\%) were started on multiple new antihypertensives (supplementary figure B). Additionally, 628 (4\%) patients were discharged on an increased dose of at least one antihypertensive.

Both elevated inpatient and outpatient blood pressures were predictive of receipt an antihypertensive intensification, with a stronger association for inpatient blood pressure than outpatient blood pressure (table 2). More than half of patients discharged with antihypertensive intensifications had well controlled outpatient blood pressure before admission (1082/2074; 52\%).

Probability of antihypertensive intensification by inpatient and outpatient blood pressure

Next, we calculated the rate of antihypertensive intensification after adjusting for several demographic, clinical, and other characteristics, to better understand the independent effect of outpatient and inpatient blood pressure control on this outcome. Patients with moderately elevated inpatient blood pressure had a $25 \%$ (95\% confidence interval 23\% to $78 \%$ ) probability of antihypertensive intensification. Similarly, patients with severely elevated inpatient blood pressure had 


\begin{tabular}{|c|c|c|c|}
\hline Blood pressure category & Intensification, No (\%) & \multicolumn{2}{|l|}{ Odds ratio $(95 \% \mathrm{CI})$} \\
\hline Overall cohort $(n=14915)$ & $2074(13.9)$ & & \\
\hline \multicolumn{4}{|l|}{ Outpatient blood pressure*: } \\
\hline Well controlled $(n=9636)$ & $1082(11.2)$ & 1 (reference) & 1 (reference) \\
\hline Very high $(n=287)$ & $92(32.1)$ & $3.73(2.89$ to 4.82$)$ & $3.28(2.09$ to 5.14$)$ \\
\hline \multicolumn{4}{|l|}{ Inpatient blood pressuret: } \\
\hline Not elevated $(n=11218)$ & $985(8.8)$ & 1 (reference) & 1 (reference) \\
\hline Moderately elevated $(n=2755)$ & $718(26.1)$ & $3.66(3.29$ to 4.08$)$ & $3.54(3.03$ to 4.12$)$ \\
\hline Severely elevated $(n=775)$ & $349(45.0)$ & $8.51(7.28$ to 9.95$)$ & $7.67(5.91$ to 9.95$)$ \\
\hline
\end{tabular}

a $42 \%$ (38\% to $47 \%$ ) probability of antihypertensive intensification. Neither rate varied significantly across patients with better or worse levels of outpatient blood pressure control $(\mathrm{P}=0.35$ for difference in severely elevated inpatient blood pressure group and $\mathrm{P}=0.05$ for difference in moderately elevated inpatient blood pressure group; fig 2). Among patients with previously

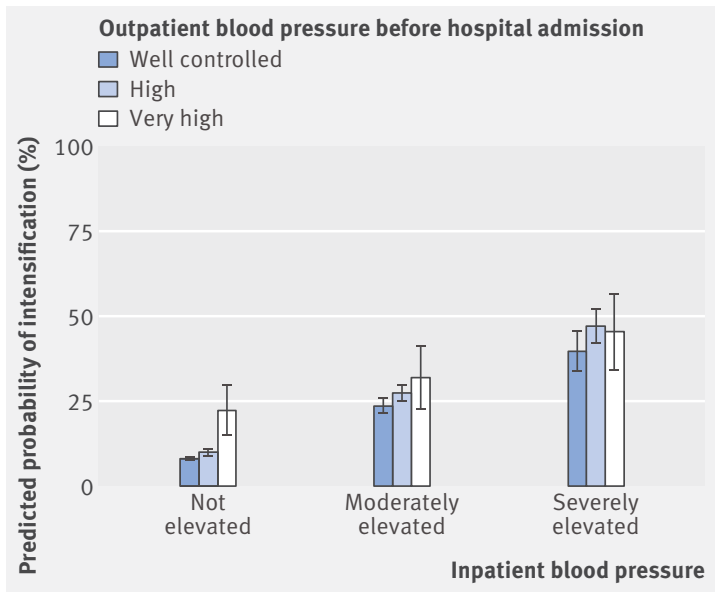

Fig 2 | Predicted probability of antihypertensive intensification by inpatient and outpatient blood pressure (BP). Error bars indicate $95 \% \mathrm{Cl}$. Outpatient $B P$ control measured using median of three outpatient BPs before admission and defined as well controlled if systolic blood pressure (SBP) $<140 \mathrm{~mm} \mathrm{Hg}$, high if SBP 140-179 mm Hg or diastolic blood pressure (DBP) 90-100 $\mathrm{mm} \mathrm{Hg}$, or very high if $S B P \geq 180 \mathrm{~mm} \mathrm{Hg}$ or DBP $>100 \mathrm{~mm}$ $\mathrm{Hg}$. Inpatient BP control defined by number of elevated $B P$ recordings and defined as severely elevated if $\geq 3$ recordings of $S B P>180 \mathrm{~mm} \mathrm{Hg}$, moderately elevated if $\geq 3$ recordings of $S B P>160 \mathrm{~mm} \mathrm{Hg}$ without meeting criteria for severely elevated, or not elevated. Predicted probabilities estimated following mixed effect logistic regression accounting for age category, sex, ethnicity, income, Charlson comorbidity index, length of stay, primary discharge diagnosis, year, hospital training status, inpatient BP, outpatient BP, an interaction term for inpatient and outpatient BP, and random effects to account for clustering by Veterans Affairs hospital well controlled outpatient blood pressure, 8\% (95\% confidence interval $7 \%$ to $9 \%$ ) of patients without elevated inpatient blood pressure, $24 \%$ (21\% to $26 \%$ ) of patients with moderately elevated inpatient blood pressure, and $40 \%$ (34\% to $46 \%$ ) of patients with severely elevated inpatient blood pressure were discharged with intensified antihypertensive regimens. Patients whose inpatient blood pressure was not elevated had a $9 \%$ ( $8 \%$ to $10 \%)$ probability of antihypertensive intensification. The small minority of this group (115/10 936) who had very high outpatient blood pressure were more likely to be intensified than those with normal or high outpatient blood pressure $(\mathrm{P}<0.001$; fig 2).

\section{Probability of antihypertensive intensification by comorbidity and life expectancy}

In adjusted analyses, we found no significant differences in predicted probability of antihypertensive intensification at discharge by life expectancy $(\mathrm{P}=0.07)$, diagnosis of dementia $(\mathrm{P}=0.95)$, or metastatic malignancy ( $\mathrm{P}=0.13$ ) (fig 3, top). Patients with a previous history of congestive heart failure had a $2 \%$ $(0.4 \%$ to $4 \%)$ increased probability of antihypertensive intensification compared with patients without (fig 3, bottom). No difference existed in the probability of intensification for patients with a previous history of myocardial infarction $(\mathrm{P}=0.53)$, cerebrovascular disease $(\mathrm{P}=0.37)$, or renal disease $(\mathrm{P}=0.73)$.

\section{Sensitivity analyses}

In a sensitivity analysis excluding the 2104 patients with a secondary discharge diagnosis of congestive heart failure, 13\% (1647/12811) of the remaining patients were discharged with an antihypertensive intensification. In analyses excluding patients with a history of acute myocardial infarction or congestive heart failure, $12 \%(1239 / 10031)$ of the remaining patients were discharged with an antihypertensive intensification. In analyses including separate indicator variables for diagnoses of congestive heart failure and acute myocardial infarction, having congestive heart 


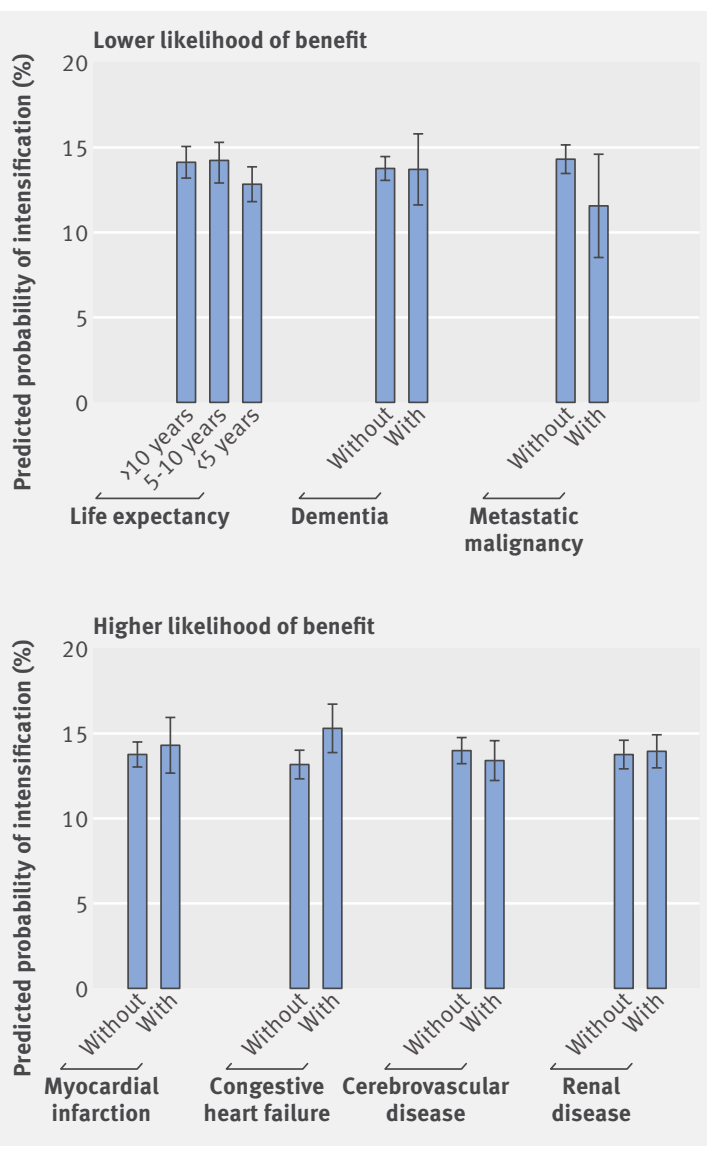

Fig 3 | Predicted probability of antihypertensive intensification by lower and higher likelihood to benefit from strict blood pressure (BP) control. Top: groups with lower likelihood of benefit. $P$ values for comparisons: life expectancy $P=0.07$, history of dementia $P=0.95$, and history of metastatic malignancy $P=0.13$. Bottom: groups with higher likelihood of benefit. $P$ values for comparison: history of myocardial infarction $P=0.53$, congestive heart failure $P=0.01$, cerebrovascular disease $P=0.37$, and renal disease $P=0.73$. Predicted probabilities for comorbidities estimated following mixed effect logistic regression accounting for age category, sex, ethnicity, income, length of stay, Charlson comorbidity indicators, primary discharge diagnosis, year, hospital training status, inpatient $B P$, outpatient BP, an interaction term for inpatient and outpatient $B P$, and random effects to account for clustering by Veterans Affairs hospital. Predicted probabilities by life expectancy generated with similar models but replacing age and Charlson comorbidity indicators with a categorical life expectancy variable calculated from age and number of comorbidities. Error bars indicate $95 \% \mathrm{Cl}$

failure was a significant predictor of receiving an antihypertensive intensification (adjusted odds ratio $1.55,95 \%$ confidence interval 1.29 to 1.74 ) but having history of acute myocardial infarction was not (1.06, -0.91 to 1.24$)$. Separately adjusting for congestive heart failure and acute myocardial infarction did not substantively affect the relation between receiving an intensification and other covariates, so we present only the results of the primary analysis.

\section{Discussion}

In a national cohort of older veterans with hypertension who were admitted to hospital for common, noncardiovascular medical conditions, we found that one in seven patients were discharged with intensifications of their antihypertensive drugs, most of whom had well controlled blood pressure before admission. Elevated inpatient blood pressure readings were a stronger predictor of intensification than were outpatient blood pressure readings. Patients with limited life expectancy, dementia, or metastatic malignancy were no less likely to receive intensifications than all other patients, despite having a decreased likelihood of clinical benefit from tight blood pressure control. Patients with history of myocardial infarction or cerebrovascular disease were no more likely to receive antihypertensive intensifications, despite having the highest likelihood of clinical benefit. Our findings indicate that decisions to discharge patients with intensified antihypertensive regimens are likely driven by inpatient blood pressure readings and not the overall context of older adults' health or long term disease control.

\section{Implications of findings}

Inpatient clinicians are faced with a surplus of routinely collected clinical data that may not always improve clinical decision making. Telemetry and daily blood draws have been identified as areas of routine data collection that are often overused. Hospital inpatients have blood pressure recordings taken multiple times a day, a practice that may assist physicians in detecting clinically important hypotension. Although elevated blood pressure is common in inpatients, ${ }^{20}$ little evidence exists to guide clinicians managing elevated inpatient blood pressure. ${ }^{21}$ National guidelines on management of hypertension do not discuss the management of inpatient hypertension in the absence of hypertensive emergencies or blood pressure sensitive acute conditions such as acute myocardial infarction and acute ischemic stroke. ${ }^{22}$

The decision to intensify antihypertensives during hospital admission may be a result of applying outpatient blood pressure guidelines to inpatient recordings. However, many factors may contribute to transiently elevated inpatient blood pressure, including acute pain, stress, anxiety, and exposure to new drugs. White coat hypertension, in which blood pressure measured during medical office visits is routinely higher than out of office blood pressure, is a well documented phenomenon. ${ }^{23}$ Patients with white coat hypertension have comparable cardiovascular risk to normotensive patients. ${ }^{2425}$ Few studies have examined the presence of a white coat effect for patients admitted to hospital, although recent research suggests that inhospital blood pressure in clinically stable patients is often higher than blood pressure measured at home after discharge. ${ }^{25}$ Our study adds to our understanding of inpatient blood pressure readings, as we found that nearly half of patients with multiple elevated inpatient blood pressure readings had well controlled outpatient blood pressure before admission. 
In some circumstances, hospital admission may be an opportunity to improve chronic disease management. ${ }^{22}$ Patients with poor outpatient blood pressure control may benefit from hospital resources, including counseling from pharmacists and nutritionists, and treatment intensification may be appropriate for certain patients when their long term disease control and health status are considered. However, our findings that patients' comorbidities and life expectancy did not influence rates of intensification suggest that current treatment practices may focus on treating the number rather than treating the patient. Our study was conducted in the Veterans Affairs health system, which, as the largest integrated health system in the US with a national electronic health record, provides inpatient clinicians with longitudinal information on blood pressure and outpatient clinical notes. As this information may not be easily accessible in other healthcare systems, we speculate that outpatient blood pressure control may be even less likely to factor into clinical decision making in other settings. Although we found that patients with congestive heart failure were more likely to receive antihypertensive intensifications, this is likely driven by Veterans Affairs' quality improvement initiatives aimed at standardizing drug regimens for heart failure, as we observed no difference in other patients likely to benefit from strict blood pressure control.

Hospital admission is also a high risk time for the adjustment of drugs. Patients, particularly older adults, may be at higher risk of falls, orthostatic hypotension, and adverse drug interactions while being treated for and recovering from acute illness. ${ }^{2627}$ Patients are often started on multiple drugs for different acute and chronic conditions, ${ }^{1-3}$ which may lead to confusion and increased risk of medication misuse and non-adherence. As the known benefits of blood pressure control come from successful long term treatment, the safest strategy for inpatient clinicians may be to routinely communicate the findings of elevated inpatient blood pressure to outpatient providers for further management after discharge rather than intensifying antihypertensives at discharge. If additional antihypertensives are given during hospital admission to control severely elevated inpatient blood pressure, clinicians (or pharmacist or nursing led medication reconciliation programs) could improve care by routinely reviewing patients' previous outpatient blood pressure readings and drug use to better inform decision about whether patients are likely to benefit from being discharged with intensified antihypertensives. This approach may also be applied to other drug treatment started during hospital admission (such as drugs for insomnia or gastric ulcer protection) that may not routinely need to be continued on discharge. Little information is available about the long term consequences of intensifying treatment for hypertension or other chronic diseases during hospital stays. Although more research is needed, on the basis of available data little rationale seems to exist for intensification of treatment based on inpatient levels for patients who have well controlled outpatient blood pressure and no acute condition that mandates short term tight blood pressure control

\section{Limitations of study}

Our analyses have several limitations. Pharmacy records may miss drugs obtained outside of the Veterans Affairs; however, we constructed our cohort to include patients receiving regular Veterans Affairs care and likely to obtain drugs from Veterans Affairs pharmacies. Furthermore, we used clinical chart review to validate pharmacy records with a high specificity and moderate sensitivity. Secondly, owing to limitations of pharmacy records, we were unable to reliably assess the frequency of drug discontinuations, leaving the possibility that some patients with at least one intensification also had other antihypertensives stopped or reduced. However, our chart review suggests that this was uncommon. Thirdly, antihypertensives may be intensified for indications other than hypertension; however, we constructed our cohort to include patients with a primary discharge diagnosis for which antihypertensives are not routinely indicated, we excluded patients with secondary diagnoses of events that may occur during hospital admission and warrant treatment with antihypertensives, such as atrial fibrillation and acute myocardial infarction, and our results remained similar in sensitivity analyses that excluded patients with coexisting congestive heart failure. Fourthly, the Veterans Affairs patient population is primarily male, which may limit generalizability. However, as our study examined physicians' practices and the Veterans Affairs is a primary training site for many graduate medical education programs, practices developed by trainees in the Veterans Affairs system may shape care delivery in other hospital settings in the US.

\section{Conclusions}

Despite a lack of data to guide inpatient blood pressure management, one in seven older adults with hypertension were discharged with intensifications of their antihypertensive drugs after admission to hospital for unrelated medical conditions. Decisions to intensify antihypertensives at discharge from hospital seem to be driven by inpatient blood pressure measurements and not the overall context of older adults' health or long term disease control. Further research to inform clinical practice guidelines on inpatient blood pressure management is warranted and should target understanding the relation between inpatient and outpatient blood pressure and identifying the benefits and harms of intensifying antihypertensive drugs during hospital stays. Just as outpatient hypertension guidelines recommend personalizing management to account for patients' clinical status and contextual factors, a shift away from treating all high numbers and toward a patient centered approach to inpatient blood pressure management is urgently needed.

Contributors: TSA and MAS were responsible for the study concept and design. MAS obtained funding and supervised the study. All 
authors were involved in the acquisition, analysis, or interpretation of data. TSA, BJ, KF, and YS did the statistical analysis. SN, EX, and MAS provided administrative, technical, or material support. TSA drafted the manuscript, and all authors critically revised it for important intellectual content. The corresponding author attests that all listed authors meet authorship criteria and that no others meeting the criteria have been omitted. TSA and MAS had full access to all the data in the study and are the guarantors.

Funding: MAS was supported by grants from the National Institute of Aging (K24AG049057 and P3OAG044281). TSA was supported by a national research service award training grant (NRSA T32HP19025-14-00) to the University of California, San Francisco. The funding organizations had no role in the design and conduct of the study; collection, management, analysis, and interpretation of the data; preparation or approval of the manuscript; or the decision to submit the manuscript for publication. The views expressed herein are those of the authors and do not necessarily represent the views of the US Department of Veterans Affairs or the University of California, San Francisco.

Competing interests: All authors have completed the ICMJE uniform disclosure form at www.icmje.org/coi_disclosure.pdf (available on request from the corresponding author) and declare: grant support from the US National Institute of Aging and US Health Resources and Services Administration for the submitted work; no financial relationships with any organizations that might have an interest in the submitted work in the previous three years; no other relationships or activities that could appear to have influenced the submitted work.

Ethical approval: This research was approved by the institutional review boards of the San Francisco Veteran Affairs Medical Center and the University of California, San Francisco (\#16-19904).

Data sharing: No additional data are available owing to a data use agreement with the US Department of Veterans Affairs. The Stata code used in programming or analysis can be made freely available to others.

Transparency statement: The lead authors (the manuscript's guarantors) affirm that this manuscript is an honest, accurate, and transparent account of the study being reported; that no important aspects of the study have been omitted; and that any discrepancies from the study as planned (and, if relevant, registered) have been explained.

This is an Open Access article distributed in accordance with the Creative Commons Attribution Non Commercial (CC BY-NC 4.0) license, which permits others to distribute, remix, adapt, build upon this work non-commercially, and license their derivative works on different terms, provided the original work is properly cited and the use is noncommercial. See: http://creativecommons.org/licenses/by-nc/4.0/.

1 Unroe KT, Pfeiffenberger T, Riegelhaupt S, Jastrzembski J, Lokhnygina Y, Colón-Emeric C. Inpatient medication reconciliation at admission and discharge: A retrospective cohort study of age and other risk factors for medication discrepancies. Am J Geriatr Pharmacother 2010;8:115-26. doi:10.1016/jamjopharm.2010.04.002

2 Mansur N, Weiss A, Beloosesky Y. Relationship of inhospital medication modifications of elderly patients to postdischarge medications, adherence, and mortality. Ann Pharmacother 2008;42:783-9. doi:10.1345/aph.1L070

3 Harris CM, Sridharan A, Landis R, Howell E, Wright S. What happens to the medication regimens of older adults during and after an acute hospitalization? J Patient Saf 2013;9:150-3. doi:10.1097/PTS.0b013e318286f87d

4 Kwan JL, Lo L, Sampson M, Shojania KG. Medication reconciliation during transitions of care as a patient safety strategy: a systematic review. Ann Intern Med 2013;158:397-403. doi:10.7326/0003-4819-158-5-201303051-00006

5 Coleman EA, Smith JD, Raha D, Min SI. Posthospital medication discrepancies: prevalence and contributing factors. Arch Intern Med 2005;165:1842-7. doi:10.1001/archinte.165.16.1842

6 Steinman MA, Auerbach AD. Managing chronic disease in hospitalized patients. JAMA Intern Med 2013;173:1857-8. doi:10.1001/jamainternmed.2013.9511

7 Krähenbühl-Melcher A, Schlienger R, Lampert M, Haschke M, Drewe J, Krähenbühl S. Drug-related problems in hospitals a review of the recent literature. Drug Saf 2007;30:379-407. doi:10.2165/00002018-200730050-00003
8 Gray SL, Mahoney JE, Blough DK. Adverse drug events in elderly patients receiving home health services following hospital discharge. Ann Pharmacother 1999:33:1147-53 doi:10.1345/aph.19036

9 Kansagara D, Fu R, Freeman M, Wolf F, Helfand M. Intensive insulin therapy in hospitalized patients: a systematic review. Ann Intern Med 2011;154:268-82. doi:10.7326/0003-4819-154-4-201102150-00008

10 Axon RN, Cousineau L, Egan BM. Prevalence and management of hypertension in the inpatient setting: a systematic review. J Hosp Med 2011;6:417-22. doi:10.1002/jhm.804

11 Forster AJ, Murff HJ, Peterson JF, Gandhi TK, Bates DW. The incidence and severity of adverse events affecting patients after discharge from the hospital. Ann Intern Med 2003;138:161-7. doi:10.7326/0003-4819-138-3-200302040-00007

12 Forster AJ, Clark HD, Menard A, et al. Adverse events among medical patients after discharge from hospital. CMAI 2004:170:345-9.

13 Healthcare Cost and Utilization Project. Clinical Classification Software (CCS) for ICD-9-CM. 2017. http://www.hcup-us.ahrq.gov/ toolssoftware/ccs/ccs.jsp.

14 Szeto HC, Coleman RK, Gholami P, Hoffman BB, Goldstein MK Accuracy of computerized outpatient diagnoses in a Veterans Affairs general medicine clinic. Am J Manag Care 2002;8:37-43.

15 Chobanian AV, Bakris GL, Black HR, et al, Joint National Committee on Prevention, Detection, Evaluation, and Treatment of High Blood Pressure, National Heart, Lung, and Blood InstituteNational High Blood Pressure Education Program Coordinating Committee. Seventh report of the Joint National Committee on Prevention, Detection, Evaluation, and Treatment of High Blood Pressure. Hypertension 2003;42:1206-52. doi:10.1161/01.HYP.0000107251.49515.c2

16 Goedken AM, Lund BC, Cook EA, Schroeder MC, Brooks JM. Application of a framework for determining number of drugs. BMC Res Notes 2016;9:272. doi:10.1186/s13104-016-2076-5

17 Choudhry NK, Shrank WH, Levin RL, et al. Measuring concurrent adherence to multiple related medications. Am J Manag Care 2009; 15:457-64.

18 Deyo RA, Cherkin DC, Ciol MA. Adapting a clinical comorbidity index for use with ICD-9-CM administrative databases. / Clin Epidemiol 1992;45:613-9. doi:10.1016/0895-4356(92)90133-8

19 Sussman JB, Kerr EA, Saini SD, et al. Rates of Deintensification of Blood Pressure and Glycemic Medication Treatment Based on Levels of Control and Life Expectancy in Older Patients With Diabetes Mellitus. JAMA Intern Med 2015;175:1942-9. doi:10.1001/jamainternmed.2015.5110

20 Axon RN, Cousineau L, Egan BM. Prevalence and management of hypertension in the inpatient setting: a systematic review. J Hosp Med 2011;6:417-22. doi:10.1002/jhm.804

21 Weder AB. Treating acute hypertension in the hospital: a Lacuna in the guidelines. Hypertension 2011:57:18-20. doi:10.1161/HYPERTENSIONAHA.110.164194

22 Whelton PK, Carey RM, Aronow WS, et al. 2017 ACC/AHA/AAPA/ ABC/ACPM/AGS/APhA/ASH/ASPC/NMA/PCNA Guideline for the Prevention, Detection, Evaluation, and Management of High Blood Pressure in Adults: A Report of the American College of Cardiology/American Heart Association Task Force on Clinica Practice Guidelines. I Am Coll Cardiol 2018;71:e127-248. doi:10.1016/j.jacc.2017.11.006

23 Franklin SS, Thijs L, Asayama K, et al, IDACO Investigators. The cardiovascular risk of white-coat hypertension. I Am Coll Cardiol 2016;68:2033-43. doi:10.1016/j.jacc.2016.08.035

24 Cappelleri C, Janoschka A, Berli R, et al. Twenty-four-hour ambulatory blood pressure monitoring in very elderly patients: Comparison of in-hospital versus home follow-up results. Medicine (Baltimore) 2017;96:e7692 doi:10.1097/MD.0000000000007692

25 Blecker S, Meisel T, Dickson VV, Shelley D, Horwitz LI. "We're Almost Guests in Their Clinical Care": Inpatient Provider Attitudes Toward Chronic Disease Management. J Hosp Med 2017;12:162-7. doi:10.12788/jhm.2699

26 Leape LL, Brennan TA, Laird N, et al. The nature of adverse events in hospitalized patients. Results of the Harvard Medical Practice Study II. N Engl I Med 1991;324:377-84. doi:10.1056/NEJM199102073240605

27 Thomas EJ, Brennan TA. Incidence and types of preventable adverse events in elderly patients: population based review of medical records. $B M$ J 2000;320:741-4. doi:10.1136/bmj.320.7237.741

\section{Supplementary materials}

